\title{
Tanshinone IIA inhibits gastric carcinoma AGS cells by decreasing the protein expression of VEGFR and blocking Ras/Raf/MEK/ERK pathway
}

\author{
CHIN-CHENG SU ${ }^{1-4}$ \\ ${ }^{1}$ Tumor Research Center of Integrative Medicine, ${ }^{2}$ Comprehensive Breast Cancer Center, ${ }^{3}$ Department of Surgery, \\ Changhua Christian Hospital, Changhua 50006; ${ }^{4}$ School of Chinese Medicine, College of Chinese Medicine, \\ China Medical University, Taichung 40402, Taiwan, R.O.C.
}

Received October 22, 2015; Accepted January 15, 2018

DOI: 10.3892/ijmm.2018.3407

\begin{abstract}
The RAS/RAF/MEK/ERK pathway is one of the most frequently dysregulated kinase cascades in human cancer, that facilitate the proliferation and survival of cancers driven by growth factor receptors. Tanshinone IIA (Tan-IIA) was extracted from Danshen (Salviae Miltiorrhizae Radix). Tan-IIA inhibition of the proliferation of gastric cancer are well documented, but the molecular mechanisms of Tan-IIA inhibition of gastric cancer have not been well elucidated. We evaluated the protein expression of vascular epidermal growth factor receptor (VEGFR), human epidermal growth factor receptor 2(HER2), Ras, Raf, MEK, ERK, PARP, caspase-3 and $\beta$-actin in AGS cells by western blotting. The results showed that AGS cells treated with Tan-IIA upregulated the protein expression of PARP and caspase-3 but decreased VEGFR, HER2, Ras, Raf, MEK and ERK time- and dose-dependently. These findings demonstrated that Tan-IIA inhibited human gastric cancer AGS cells; one of the molecular mechanisms may be through decreasing the protein expression of VEGFR and HER2, then blocking the Ras/Raf/MEK/ERK pathway to induce the activation of PARP and caspase- 3 to induce apoptosis.
\end{abstract}

\section{Introduction}

Tanshinone IIA (Tan-IIA; C19H18O3), is one of the active components in Radix Salviae miltiorrhizae (1,2), with antioxidant properties $(3,4)$ and anti-inflammatory activities $(5,6)$. Tan-IIA can inhibit many human cancer cell lines through different molecular mechanisms, such as colon cancer colo205 cells (7), breast cancer MDA-MB-231 cells (8),

Correspondence to: Dr Chin-Cheng Su, Tumor Research Center of Integrative Medicine, Changhua Christian Hospital, 135 Nan-Hsiao Street, Changhua 50006, Taiwan, R.O.C.

E-mail: succ.maeva@msa.hinet.net

Key words: Tanshinone IIA, gastric carcinoma AGS cells, vascular epidermal growth factor receptor, Ras/Raf/MEK/ERK pathway non-small cell lung cancer A549 cells (9), small cell lung cancer H146 cells (10), hepatocellular carcinoma Hep-J5 cells (11), breast cancer BT-20 cells (12) and pancreatic cancer $\mathrm{BxPC}-3$ cells in vitro $(13,14)$. The transmembrane tyrosine kinase has been strongly implicated in the growth, survival, and metastasis of a wide variety of human tumors $(15,16)$. The PI3K/AKT/mTOR and RAS/RAF/MEK/extracellular signal-regulated kinase (ERK) pathways are two of the most frequently dysregulated kinase cascades in human cancer are well documented $(17,18)$. Both pathways represent important signal transduction mechanisms that facilitate the proliferation and survival of cancers driven by growth factor receptors, such as human epidermal growth factor receptor 2 (HER2) or epidermal growth factor receptor (EGFR). The individual downstream components of these signaling cascades either through somatic mutation or epigenetic modification, are also known to be frequently altered in cancer, thus contributing to tumorigenesis and resistance to anticancer therapies (19). It is well documented that Tan-IIA can inhibit the proliferation of AGS cells through inducing ER stress and intrinsic pathway to induce apoptosis (20). Tan-IIA also can inhibit human gastric cancer AGS cells through inducing G2/M phase arrest, and extrinsic pathway to induce apoptosis (21). These indicate that Tan-IIA may be one of the complementary medicines for gastric cancer. But the molecular mechanisms of Tan-IIA in gastric cancer cells remain unclear. In the present study, we investigated the protein expression levels of VEGFR, HER2, Ras, Raf, MEK, ERK, PARP and caspase-3 in human gastric cancer AGS cells treated with Tan-IIA.

\section{Materials and methods}

Tan-IIA was obtained from Sigma-Aldrich (St. Louis, MO, USA) (CAS no. 568-72-9); The HER2 (\#2165, MW 185 kDa), Ras (\#3339, MW 21 kDa), Raf (\#12552, MW 75 kDa), MEK (\#9126, MW 45 kDa), and caspase-3 (\#9661, MW 17 kDa) antibodies were all obtained from Cell Signaling Technology, Inc. (Beverly, MA, USA). VEGFR (N100-527, MW 150 kDa) antibodies were both obtained from Novus Biologicals (Littleton, CO, USA). ERK (sc-94, MW $44 \mathrm{kDa}$ ) and PARP (sc-7150, MW 116, $89 \mathrm{kDa}$ ) antibodies were all obtained from Santa Cruz 


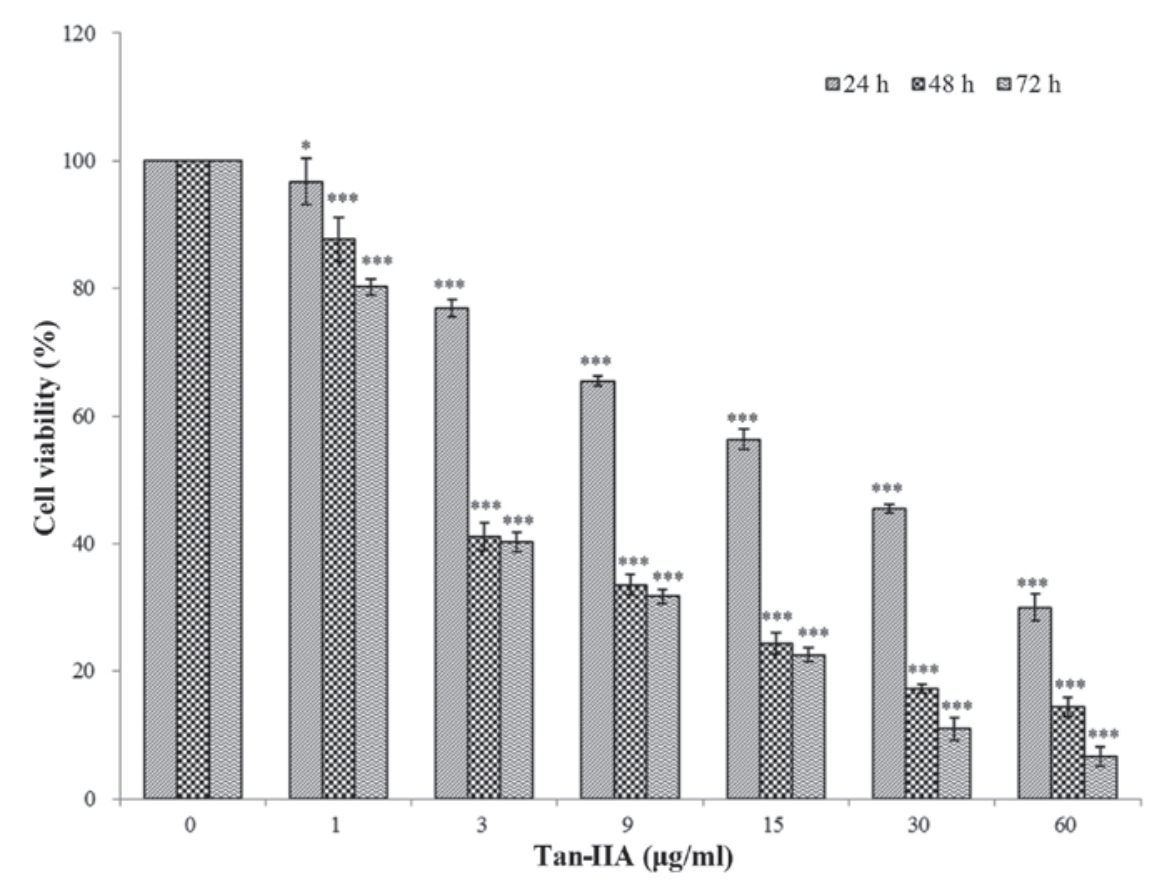

Figure 1. Cytotoxic effects of tanshinone IIA (Tan-IIA) on AGS cells. The cytotoxic effects of Tan-IIA on AGS cells were determined by MTT assay as described in Materials and methods. Each point is the mean \pm SD of 3 experiments. ${ }^{*} \mathrm{P}<0.05 ;{ }^{* *} \mathrm{P}<0.01 ;{ }^{* * *} \mathrm{P}<0.001$.

Biotechnology, Inc. (Dallas, TX, USA). F-12K medium, fetal bovine serum (FBS), penicillin-streptomycin and glutamine were obtained from Gibco-BRL (Grand Island, NY, USA). Potassium phosphate and $0.2 \mathrm{~mm}$ PVFD membranes were purchased from Merck Co. (Darmstadt, Germany); BioMax film was from Kodak (Rochester, NY, USA). Sodium deoxycholate, leupeptin, Triton X-100, Tris-HCl, ribonuclease-A, sodium pyruvate, HEPES, dimethyl sulfoxide (DMSO), 3-(4,5-dimethylthiazol-2-y1)-2,5-diphenyltetrazolium bromide (MTT) and Tween-20, mouse anti- $\beta$-actin were from SigmaAldrich. The AGS human gastric adenocarcinoma cell line (BCRC no. 60102) was obtained from the Food Industry Research and Development Institute (Hsinchu, Taiwan).

Cell culture. The human gastric adenocarcinoma AGS cells were obtained from the Food Industry Research and Development Institute. The cell culture procedure was as previously described $(20,21)$. Briefly, the AGS cells were placed into $75-\mathrm{cm}^{2}$ tissue culture flasks and maintained in F-12K contained with 10\% heat-inactivated FBS (Gibco-BRL, Grand Island, NY, USA), $100 \mathrm{U} / \mathrm{ml}$ penicillin and $100 \mu \mathrm{g} / \mathrm{ml}$ streptomycin. Cells were grown at $37^{\circ} \mathrm{C}$ in a humidified atmosphere of $95 \%$ air and $5 \% \mathrm{CO}_{2}$. All data presented are from at least three independent experiments.

Cytotoxicity assay. The cytotoxicity of Tan-IIA for AGS cells was evaluated by MTT assay in triplicate as previously described $(20,21)$. Briefly, The AGS cells were plated in 96-well plates at a density of $2 \times 10^{4}$ cells/well for 16-20 h. Thereafter, the cells were treated with various concentrations $(0,1,3,9,15,30$ and $60 \mu \mathrm{g} / \mathrm{ml})$ of Tan-IIA for 24,48 and $72 \mathrm{~h}$. Subsequently, the cells were incubated with $1 \mathrm{mg} / \mathrm{ml}$ of MTT in fresh complete F-12K medium for $1 \mathrm{~h}$. The surviving cells converted MTT to formazan by forming a blue-purple color when dissolved in dimethyl sulfoxide. The intensity of formazan was measured at $590 \mathrm{~nm}$ using a microplate reader. The relative percentage of cell viability was calculated by dividing the absorbance of treated cells by that of the control in each experiment, using the following formula: proliferation rate $(\%)=(\mathrm{OD}$ test - OD blank) $\times 100$, where OD test and OD blank are the optical density of the test substances and the blank control, respectively.

Western blot analysis. The western blot procedures followed previous reports $(20,21)$. Briefly, AGS cells were treated with various concentrations of Tan-IIA for different durations, and then the cells were lysed in ice-cold whole cell extract buffer containing the protease inhibitors. The lysate was vibrated for $30 \mathrm{~min}$ at $4^{\circ} \mathrm{C}$ and centrifuged at $12,281 \mathrm{x} \mathrm{g}$ for $10 \mathrm{~min}$. Protein concentration was measured by BCA protein assay kit (Pierce, Rockford, IL, USA). Equal amounts of proteins were subjected to electrophoresis using $12 \%$ sodium dodecyl sulfate-polyacrylamide gels. To verify equal protein loading and transfer, proteins were then transferred to polyvinylidene difluoride membranes and the membranes were blocked for $1 \mathrm{~h}$ at $4^{\circ} \mathrm{C}$ using blocking buffer $(5 \%$ non-fat dried milk in solution containing $50 \mathrm{mM}$ Tris/ $\mathrm{HCl}(\mathrm{pH} 8.0), 2 \mathrm{mM} \mathrm{CaCl}_{2}$, $80 \mathrm{mM}$ sodium chloride, $0.05 \%$ Tween -20 and $0.02 \%$ sodium azide). The membranes were then incubated for $2 \mathrm{~h}$ at room temperature with specific primary antibody followed by anti-rabbit or anti-mouse immunoglobulin G-horseradish peroxidase conjugated secondary antibodies. The membranes were washed three times for $10 \mathrm{~min}$ with washing solution. Finally, the protein bands were visualized on the X-ray film using the enhanced chemiluminescence detection system (PerkinElmer Life and Analytical Sciences, Boston, MA, USA).

Statistical analysis. Values are presented as the means \pm SD. The Student's t-test was used to analyze statistical significance. 

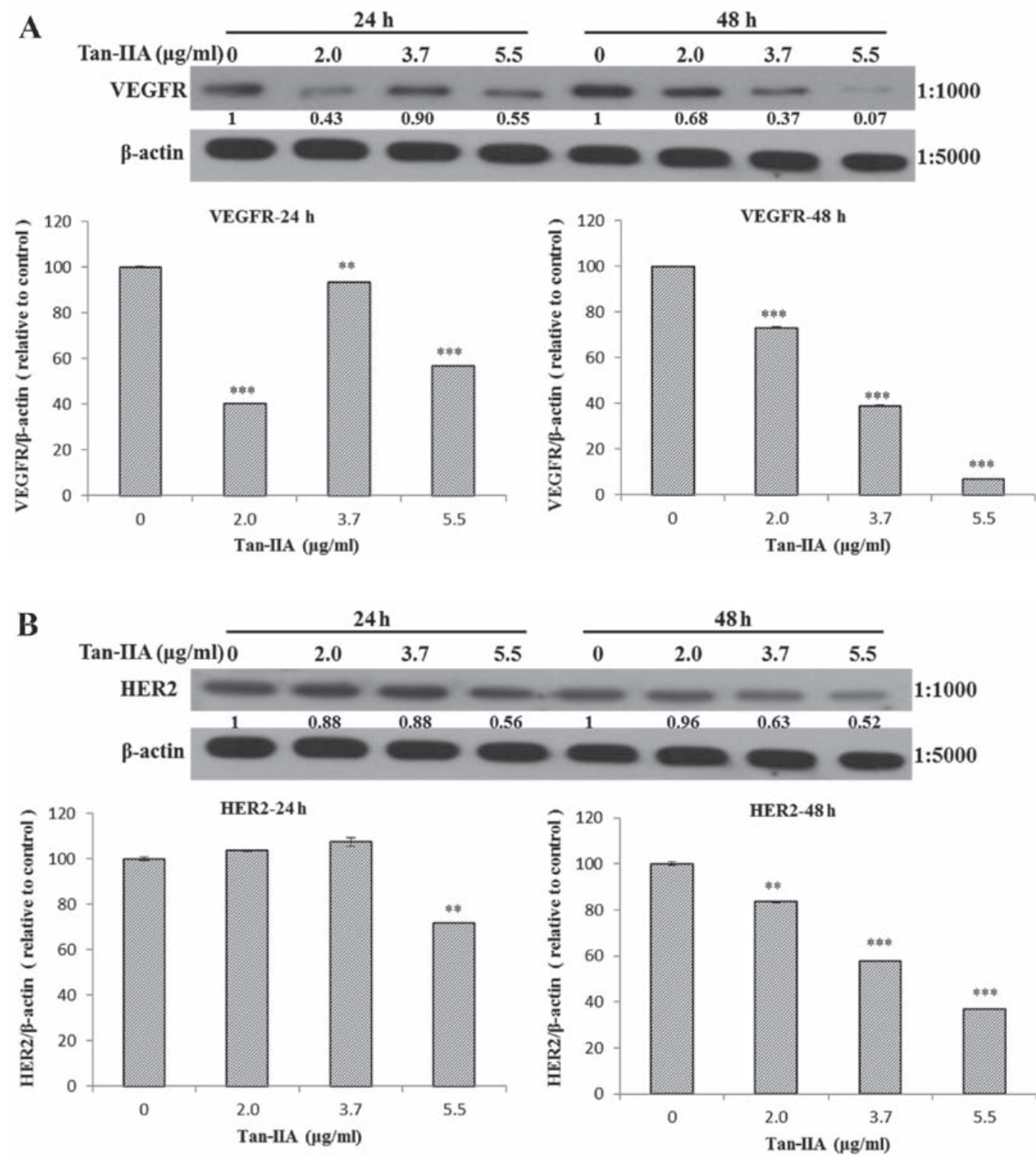

Figure 2. Protein expression of vascular epidermal growth factor receptor (VEGFR), human epidermal growth factor receptor 2 (HER2) and $\beta$-actin in AGS cells. The AGS cells were treated with various concentrations of tanshinone IIA (Tan-IIA) $(0,2.0,3.7$ and $5.5 \mu \mathrm{g} / \mathrm{ml})$ for 24 or $48 \mathrm{~h}$ and then the protein expression levels were evaluated by western blot analysis as described in Materials and methods. The results showed that Tan-IIA decreased the protein expression level of (A) VEGFR and (B) HER2 significantly. ${ }^{*} \mathrm{P}<0.05 ;{ }^{* *} \mathrm{P}<0.01 ;{ }^{* * *} \mathrm{P}<0.001$.

P-value $<0.05$, was considered to indicate a statistically significant difference for all the tests, at $\mathrm{P}<0.05, \mathrm{P}<0.01$ and $\mathrm{P}<0.001$.

\section{Results}

Effects of Tan-IIA in the viability of AGS cells. The results revealed that Tan-IIA can inhibit AGS cells in a time- and dose-dependent manner. The half-maximal inhibitory concentration $\left(\mathrm{IC}_{50}\right)$ was $5.5,3.7$ and $3.5 \mu \mathrm{g} / \mathrm{ml}$ at 24,48 and $72 \mathrm{~h}$, respectively (Fig. 1), this is in agreement with our previous studies $(20,21)$.

Effects of Tan-IIA on the protein expression of VEGFR, HER2, Ras, Raf, MEK, ERK, PARP, caspase-3 and $\beta$-actin in $A G S$ cells. The AGS cells were treated with various concentrations of Tan-IIA $(0,2.0,3.7$ and $5.5 \mu \mathrm{g} / \mathrm{ml})$ for 24 or $48 \mathrm{~h}$ and then the protein expression levels of VEGFR, HER2, Ras,
Raf, MEK, ERK, PARP, caspase- 3 and $\beta$-actin were evaluated by western blot analysis. The results showed that Tan-IIA can decrease the protein expression levels of VEGFR (Fig. 2A), HER2 (Fig. 2B), Ras (Fig. 3A), Raf (Fig. 3B), MEK (Fig. 3C) and ERK (Fig. 3D), but increase PARP (Fig. 4A) and caspase-3 (Fig. 4B) levels significantly.

Effects of Tan-IIA on the protein expression of VEGFR, HER2, Ras, Raf, MEK, ERK, PARP, caspase-3 and $\beta$-actin in AGS cells. The AGS cells were treated with Tan-IIA $(3.7 \mu \mathrm{g} / \mathrm{ml})$ for different durations $(0,24$ and $48 \mathrm{~h})$ and then the protein expression levels of VEGFR, HER2, Ras, Raf, MEK, ERK, PARP, caspase- 3 and $\beta$-actin were evaluated by western blot analysis. The results showed that Tan-IIA can decrease the protein expression levels of VEGFR (Fig. 5A), HER2 (Fig. 5B), Ras (Fig. 5C), Raf (Fig. 5D), MEK (Fig. 5E) and ERK (Fig. 5F), but increased PARP (Fig. 5G) and caspase-3 (Fig. 5H) levels significantly. 

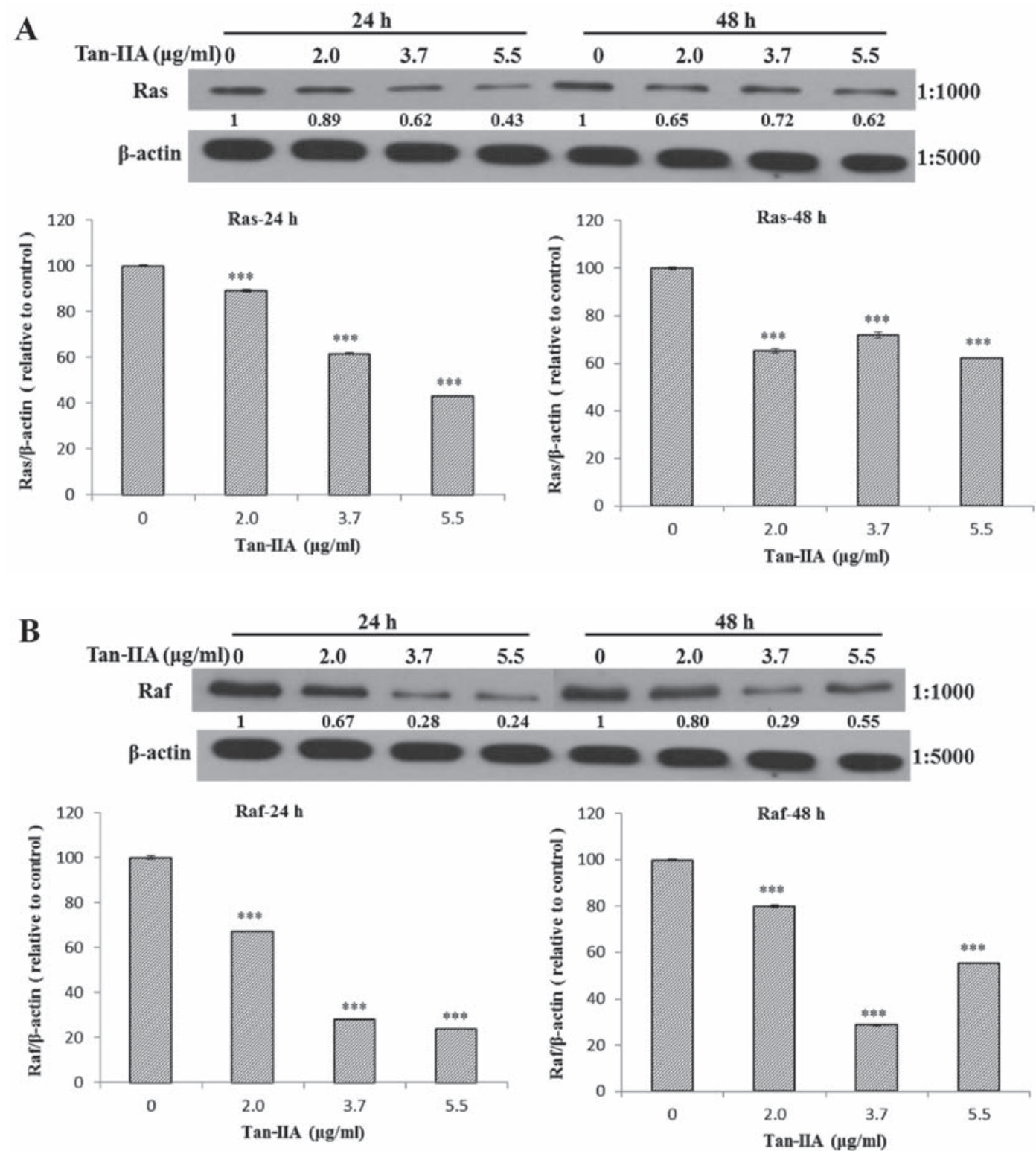

C

$24 \mathrm{~h}$

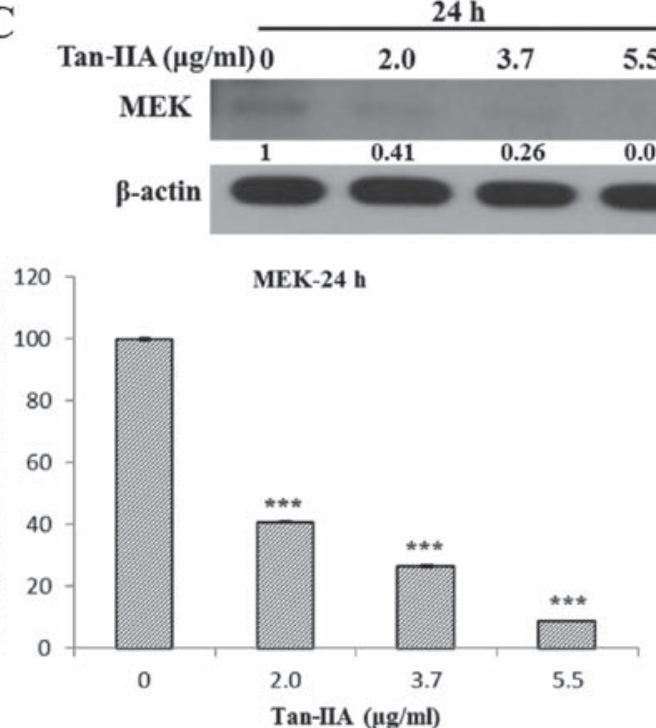

$48 \mathrm{~h}$
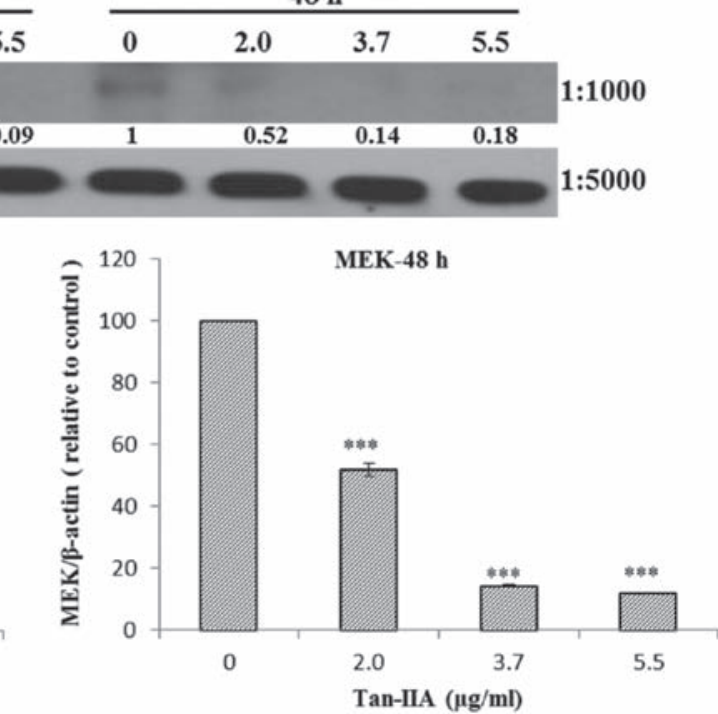

Figure 3. (A-C) Protein expression of Ras, Raf, MEK, extracellular signal-regulated kinase (ERK) and $\beta$-actin in AGS cells. The AGS cells were treated with various concentrations of tanshinone IIA (Tan-IIA) $(0,2.0,3.7$ and $5.5 \mu \mathrm{g} / \mathrm{ml}$ ) for 24 or $48 \mathrm{~h}$ and then the protein expression levels were evaluated by western blot analysis as described in Materials and methods. The results show that Tan-IIA decreased the protein expression levels of (A) Ras, (B) Raf, (C) MEK levels significantly. ${ }^{*} \mathrm{P}<0.05 ;{ }^{* *} \mathrm{P}<0.01 ;{ }^{* * *} \mathrm{P}<0.001$. 

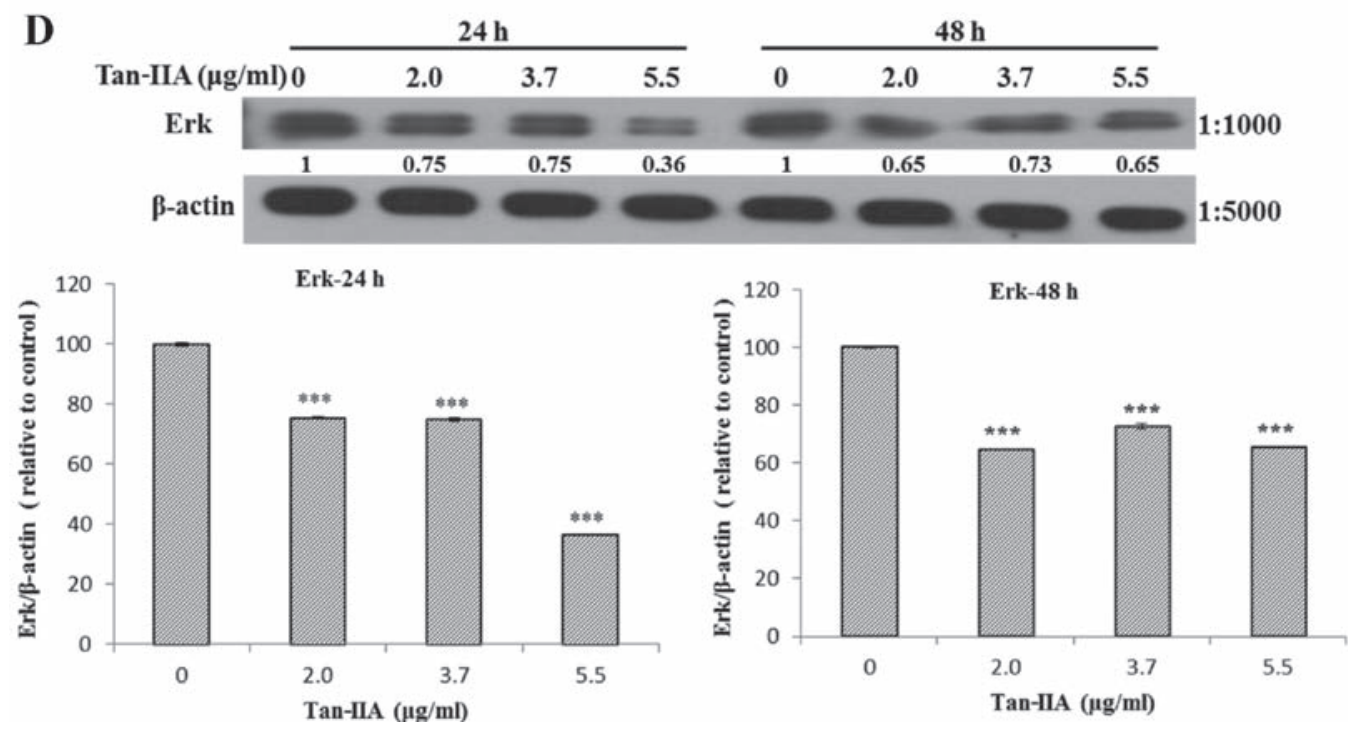

Figure 3 Continued. (D) The results showed that Tan-IIA can decrease the protein expression levels of ERK levels significantly.
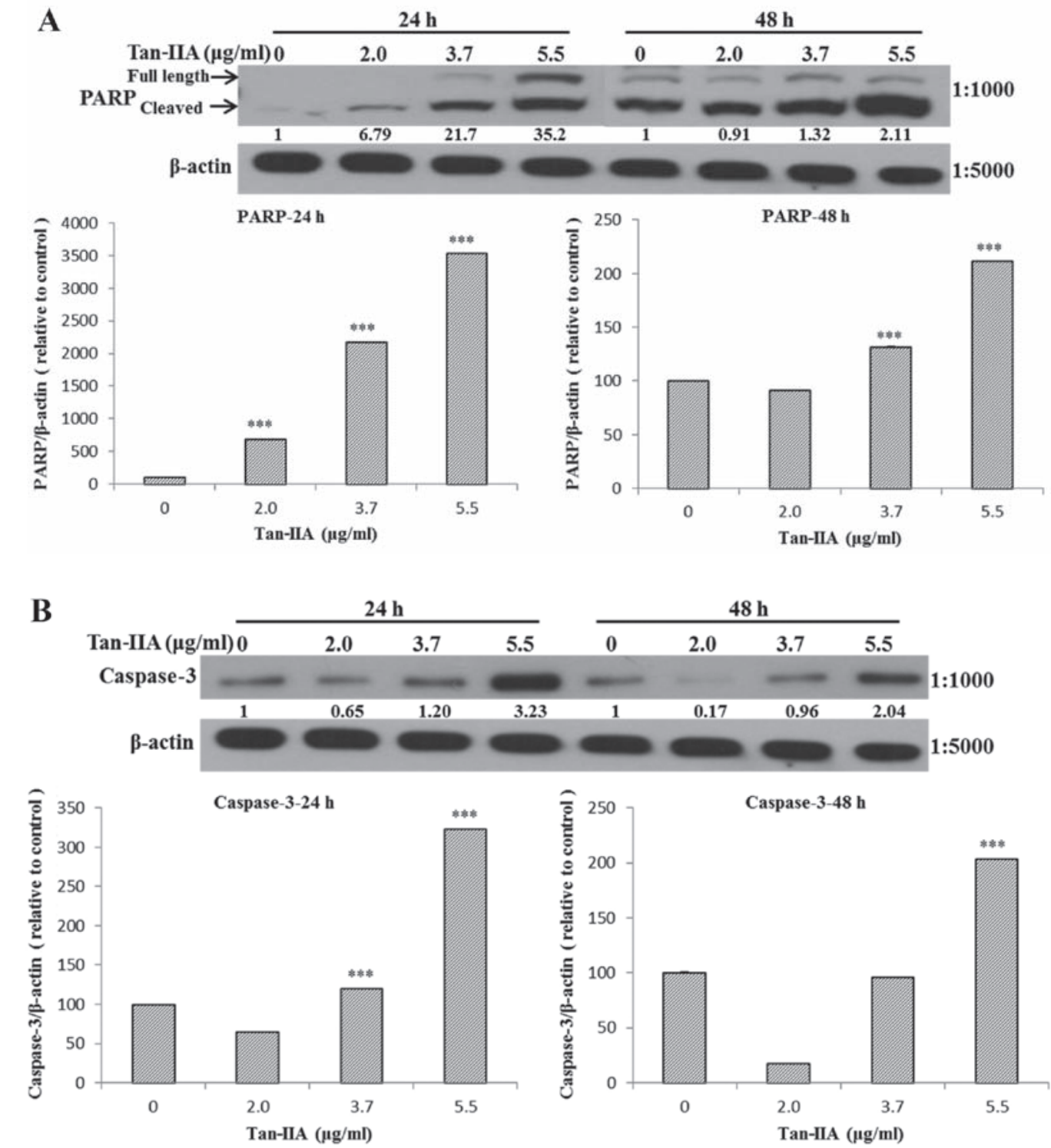

Figure 4. Protein expression of PARP, caspase-3 and $\beta$-actin in AGS cells. The AGS cells were treated with various concentrations of tanshinone IIA (Tan-IIA) $(0,2.0,3.7$ and $5.5 \mu \mathrm{g} / \mathrm{ml})$ for 24 or $48 \mathrm{~h}$ and then the protein expression levels were evaluated by western blot analysis as described in Materials and methods. The results show that Tan-IIA increased (A) PARP and (B) caspase-3 levels significantly. ${ }^{*} \mathrm{P}<0.05 ;{ }^{* * *} \mathrm{P}<0.01 ;{ }^{* * * *} \mathrm{P}<0.001$. 

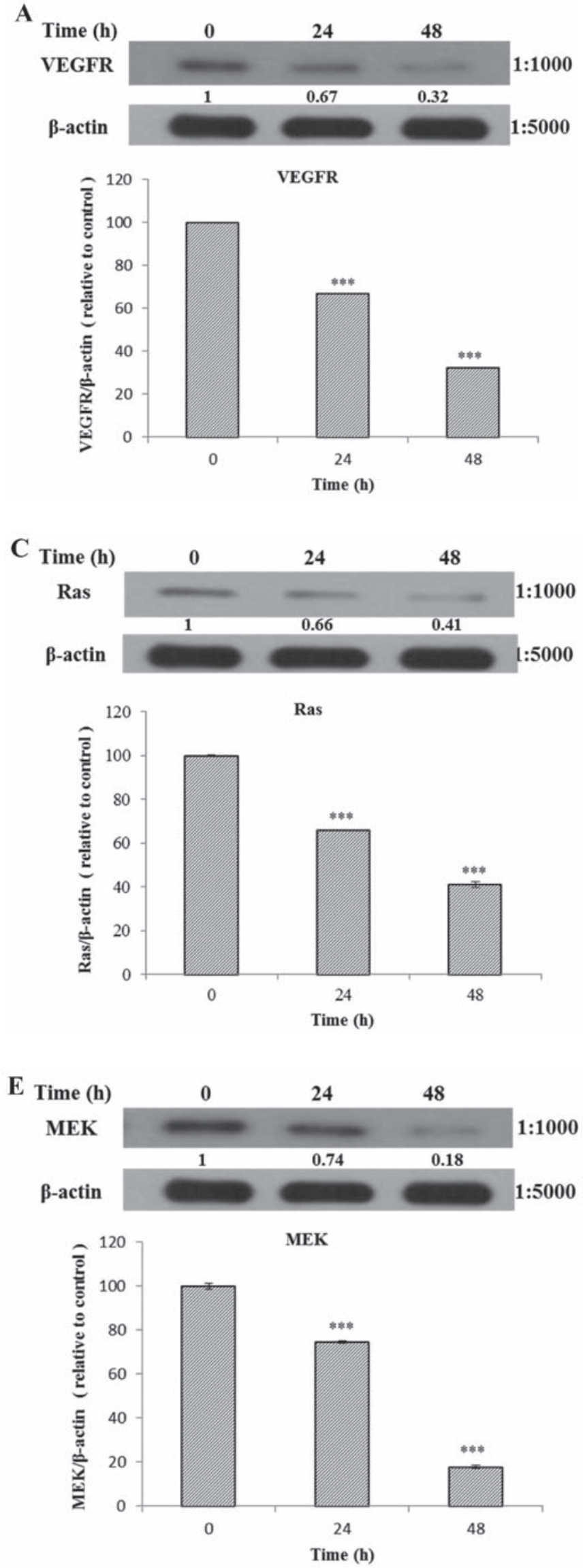
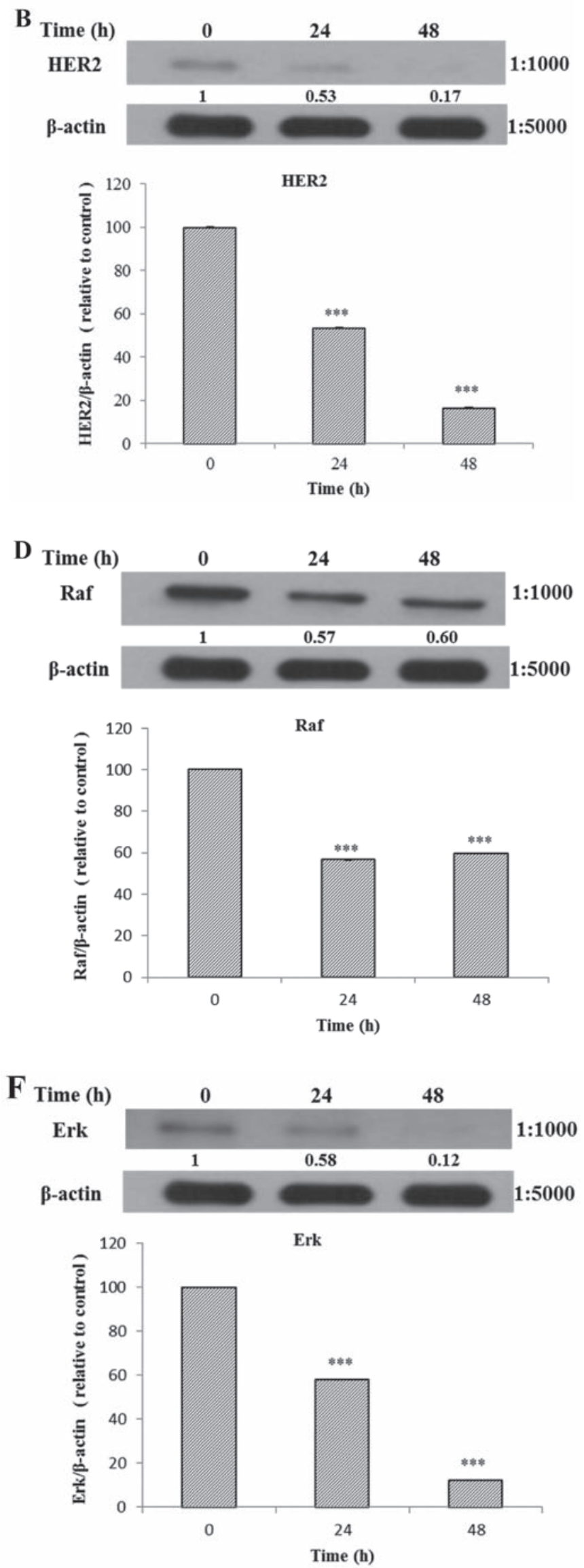

Figure 5. (A-F) Protein expression of vascular epidermal growth factor receptor (VEGFR), human epidermal growth factor receptor 2 (HER2), Ras, Raf, MEK, extracellular signal-regulated kinase (ERK), PARP, caspase-3 and $\beta$-actin in AGS cells. The AGS cells were treated with tanshinone IIA (Tan-IIA) (3.7 $\mu \mathrm{g} / \mathrm{ml})$ for different durations $(0,24$ and $48 \mathrm{~h})$ and then the protein expression levels of VEGFR, HER2, Ras, Raf, MEK, ERK, PARP, caspase-3 and $\beta$-actin were evaluated by western blot analysis. The results showed that Tan-IIA can decrease the protein expression levels of (A) VEGFR, (B) HER2, (C) Ras, (D) Raf, (E) MEK and (F) ERK levels significantly. ${ }^{*} \mathrm{P}<0.05 ;{ }^{* *} \mathrm{P}<0.01 ;{ }^{* * *} \mathrm{P}<0.001$. 

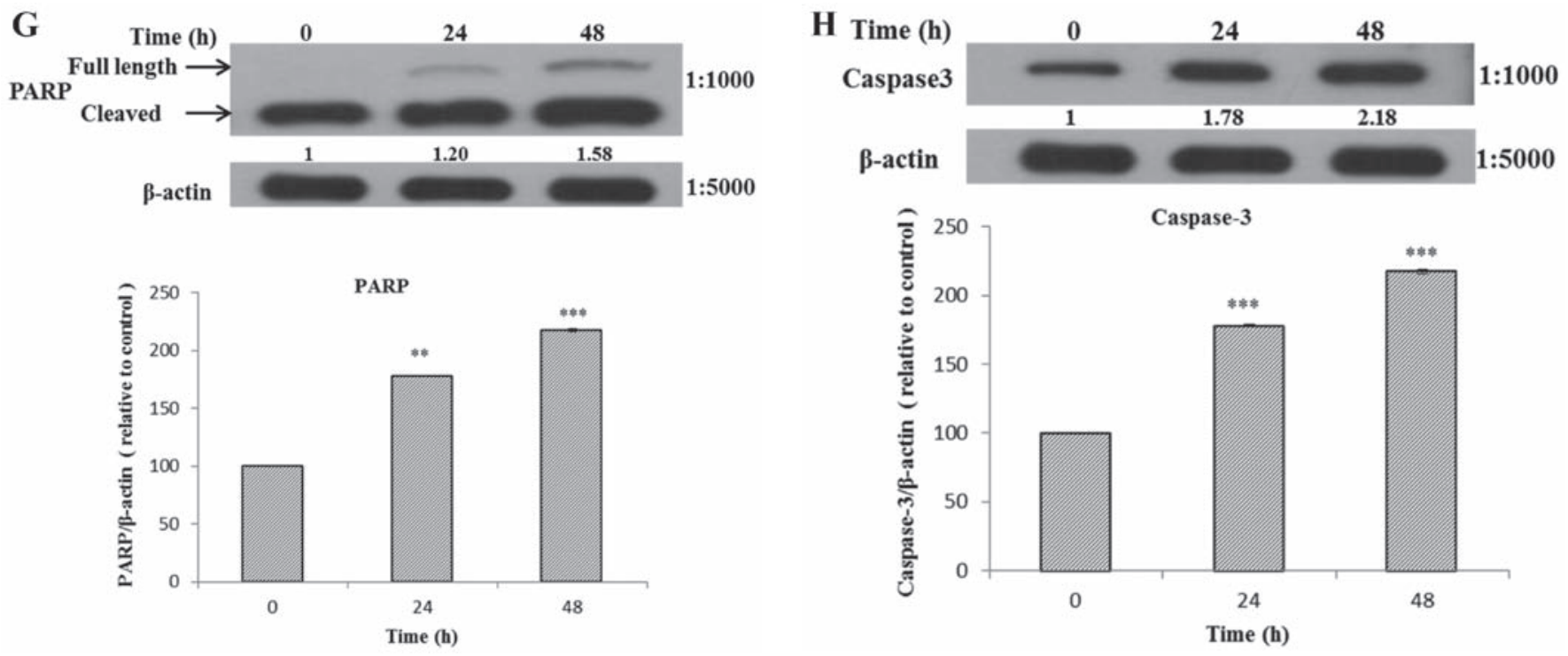

Figure 5 Continued. The results showed that Tan-IIA can increase (G) PARP and (H) caspase-3 levels significantly.

Tan-IIA

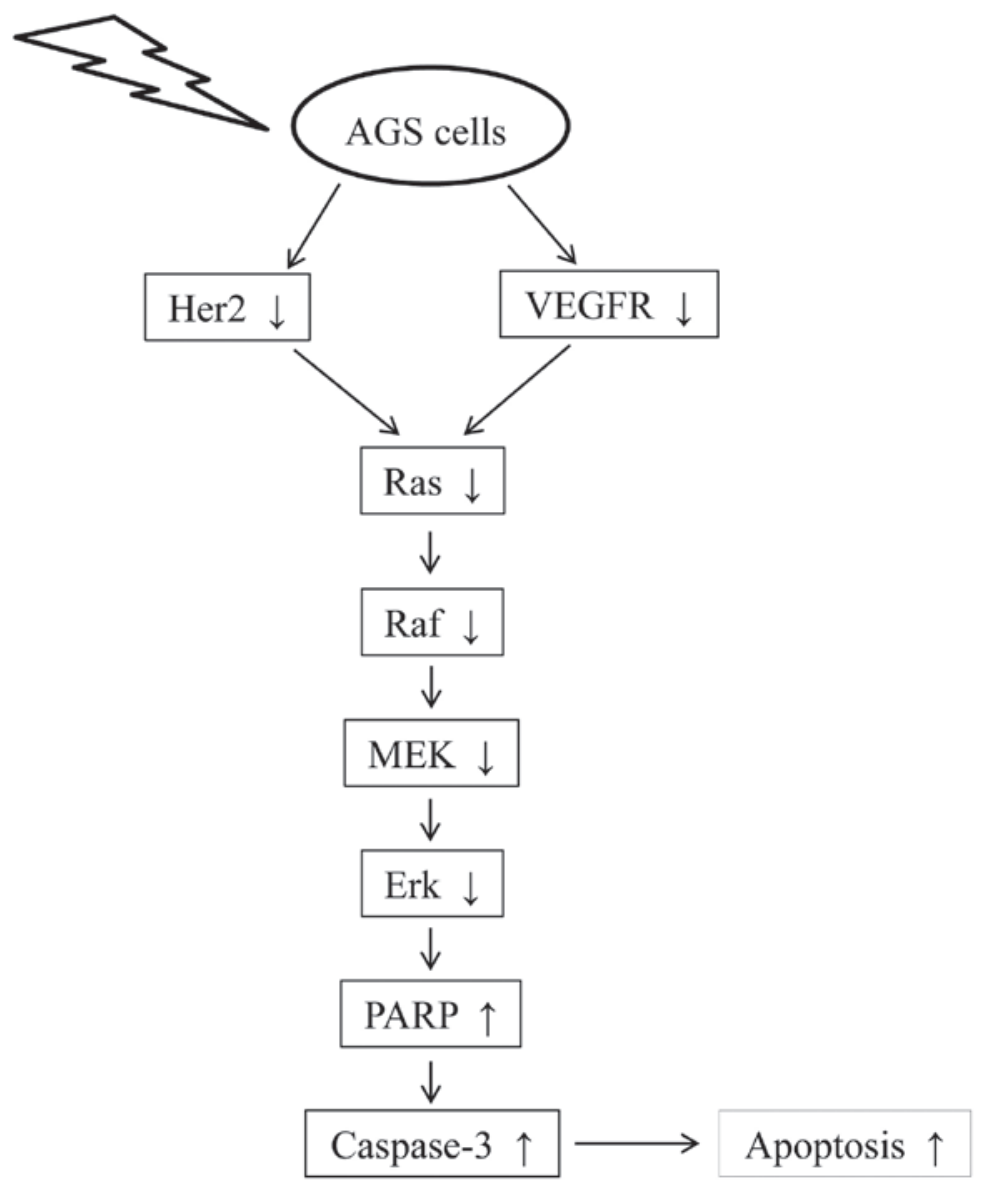

Figure 6. The proposed model for tanshinone IIA (Tan-IIA) to inhibit the proliferation of AGS cells. Tan-IIA inhibited human gastric cancer AGS cells through decreasing the protein expression of vascular epidermal growth factor receptor (VEGFR), human epidermal growth factor receptor 2 (HER2) and blocking Ras/Raf/MEK/extracellular signal-regulated kinase (ERK) pathway.

\section{Discussion}

It is well documented that Tan-IIA inhibits the proliferation of gastric cancer SGC7901 cells time- and dose-dependently through inducing apoptosis and G0/G1 phase arrest $(22,23)$. Tan-IIA also triggered the intrinsic apoptotic signaling pathway and arrested in $\mathrm{G} 2 / \mathrm{M}$ phase to inhibit the proliferation of gastric cancer MKN-45 cells (24). Our results also showed 
that Tan-IIA inhibited human gastric cancer AGS cells in a time- and dose-dependent manner in vitro.

It is well documented that Ras/Raf/MAPK pathway regulates a variety of cellular functions, and is also involved in the formation of new blood vessels, wound healing and tissue repair, cell cycle regulation, and cell migration. Ras is the most frequently mutated oncogene in human cancer. Dysregulation of Ras/Raf/MAPK pathway is a common event in cancer, signaling through this pathway is important for tumorigenesis (25-28). MAPK, which in mammalians is also called MEK, is a serine/threonine kinase activated in response to growth factors and cytokines to promote cell apoptosis and survival (29). ERK belongs to mitogen-activated protein kinase (MAPK) pathway, and is activated via phosphorylation in response to cytokines, growth factors and stress. Phospho-ERK (p-ERK) can inhibit apoptosis (30,31). Our results demonstrated that AGS cells treated with Tan-IIA can decrease the protein expression level of VEGFR, HER2, Ras, Raf, MEK, ERK, time- and dose-dependently. Our results also demonstrated that the treatment of AGS cells with Tan-IIA can increase the protein expression of PARP and caspase- 3 . These findings indicate Tan-IIA can induce apoptosis to inhibit the proliferation of AGS cells. The proposed model for tan-IIA to inhibit the proliferation of AGS cells is shown in Fig. 6. This is the first report that Tan-IIA could inhibit gastric carcinoma AGS cells by decreasing the protein expression of VEGFR, Her2 and blocking the Ras/Raf/MEK/ERK pathway to induce apoptosis. The chemotherapeutic potential of Tan-IIA for human gastric cancer warrants further study.

\section{Acknowledgements}

This study was supported by grant 102-CCH-IRP-066 from the Changhua Christian Hospital from the Research Section of the Changhua Christian Hospital, Changhua, Taiwan, R.O.C.

\section{Competing interests}

The author declares there is no competing interest.

\section{References}

1. Che AJ, Zhang JY, Li CH, Chen XF, Hu ZD and Chen XG Separation and determination of active components in Radix Salviae miltiorrhizae and its medicinal preparations by nonaqueous capillary electrophoresis. J Sep Sci 27: 569-575, 2004

2. Zhou L, Zuo Z and Chow MS: Danshen: An overview of its chemistry, pharmacology, pharmacokinetics, and clinical use. J Clin Pharmacol 45: 1345-1359, 2005.

3. Lin R, Wang WR, Liu JT, Yang GD and Han CJ: Protective effect of tanshinone IIA on human umbilical vein endothelial cell injured by hydrogen peroxide and its mechanism. J Ethnopharmacol 108: 217-222, 2006.

4. Wang AM, Sha SH, Lesniak W and Schacht J: Tanshinone (Salviae miltiorrhizae extract) preparations attenuate aminoglycoside-induced free radical formation in vitro and ototoxicity in vivo. Antimicrob Agents Chemother 47: 1836-1841, 2003.

5. Jang SI, Kim HJ, Kim YJ, Jeong SI and You YO: Tanshinone IIA inhibits LPS-induced NF-kappaB activation in RAW 264.7 cells: Possible involvement of the NIK-IKK, ERK1/2, p38 and JNK pathways. Eur J Pharmacol 542: 1-7, 2006.

6. Li W, Li J, Ashok M, Wu R, Chen D, Yang L, Yang H, Tracey KJ, Wang P, Sama AE, et al: A cardiovascular drug rescues mice from lethal sepsis by selectively attenuating a late-acting proinflammatory mediator, high mobility group box 1 . J Immunol 178: 3856-3864, 2007.
7. Su CC and Lin YH: Tanshinone IIA downregulates the protein expression of ErbB-2 and upregulates TNF- $\alpha$ in colon cancer cells in vitro and in vivo. Int J Mol Med 22: 847-851, 2008.

8. Su CC and Lin YH: Tanshinone IIA inhibits human breast cancer cells through increased Bax to Bcl-xL ratios. Int J Mol Med 22: 357-361, 2008.

9. Chiu TL and Su CC: Tanshinone IIA induces apoptosis in human lung cancer A549 cells through the induction of reactive oxygen species and decreasing the mitochondrial membrane potential. Int J Mol Med 25: 231-236, 2010.

10. Cheng CY and Su CC: Tanshinone IIA may inhibit the growth of small cell lung cancer $\mathrm{H} 146$ cells by up-regulating the Bax/Bcl-2 ratio and decreasing mitochondrial membrane potential. Mol Med Rep 3: 645-650, 2010.

11. Cheng CY and Su CC: Tanshinone IIA inhibits Hep-J5 cells by increasing calreticulin, caspase 12 and GADD153 protein expression. Int J Mol Med 26: 379-385, 2010.

12. Yan MY, Chien SY, Kuo SJ, Chen DR and Su CC: Tanshinone IIA inhibits BT-20 human breast cancer cell proliferation through increasing caspase 12, GADD153 and phospho-p38 protein expression. Int J Mol Med 29: 855-863, 2012.

13. Huang CY, Chiu TL, Kuo SJ, Chien SY, Chen DR and Su CC: Tanshinone IIA inhibits the growth of pancreatic cancer BxPC3 cells by decreasing protein expression of TCTP, MCL1 and Bcl-xL. Mol Med Rep 7: 1045-1049, 2013.

14. Su CC: Tanshinone IIA could inhibit pancreatic cancer BxPC-3 cells through increasing PERK, ATF6, caspase-12 and CHOP expression to induce apoptosis. J Biomed Sci Eng 8: 149-159, 2015.

15. Yuen JS and Macaulay VM: Targeting the type 1 insulin-like growth factor receptor as a treatment for cancer. Expert Opin Ther Targets 12: 589-603, 2008.

16. Chitnis MM, Yuen JS, Protheroe AS, Pollak M and Macaulay VM: The type 1 insulin-like growth factor receptor pathway. Clin Cancer Res 14: 6364-6370, 2008.

17. Santarpia L, Lippman SM and El-Naggar AK: Targeting the MAPK-RAS-RAF signaling pathway in cancer therapy. Expert Opin Ther Targets 16: 103-119, 2012.

18. Liu P, Cheng H, Roberts TM and Zhao JJ: Targeting the phosphoinositide 3-kinase pathway in cancer. Nat Rev Drug Discov 8: 627-644, 2009.

19. McCubrey JA, Steelman LS, Kempf CR, Chappell WH, Abrams SL, Stivala F, Malaponte G, Nicoletti F, Libra M, Bäsecke J, et al: Therapeutic resistance resulting from mutations in Raf/MEK/ERK and PI3K/PTEN/Akt/mTOR signaling pathways. J Cell Physiol 226: 2762-2781, 2011.

20. Su CC: Tanshinone IIA inhibits human gastric carcinoma AGS cell growth by decreasing BiP, TCTP, Mcl 1 and Bcl xL and increasing Bax and CHOP protein expression. Int J Mol Med 34: 1661-1668, 2014.

21. Su CC: Tanshinone IIA inhibits gastric carcinoma AGS cells through increasing p-p38, p-JNK and p53 but reducing p-ERK, CDC2 and cyclin B1 expression. Anticancer Res 34: 7097-7110, 2014.

22. Hou J, He J, Jin X, Hu T and Zhang Y: Study on optimisation of extraction process of tanshinone IIA and its mechanism of induction of gastric cancer SGC7901 cell apoptosis. Afr J Tradit Complement Altern Medicines 10: 456-458, 2013.

23. Xu M, Cao FL, Li NY, Liu YQ, Li YP and Lv CL: Tanshinone IIA reverses the malignant phenotype of SGC7901 gastric cancer cells. Asian Pac J Cancer Prev 14: 173-177, 2013.

24. Dong X, Dong J, Peng G, Hou X and Wu G: Growth-inhibiting and apoptosis-inducing effects of Tanshinone II A on human gastric carcinoma cells. J Huazhong Univ Sci Technolog Med Sci 27: 706-709, 2007

25. Kranenburg O, Gebbink MF and Voest EE: Stimulation of angiogenesis by Ras proteins. Biochim Biophys Acta 1654: 23-37, 2004.

26. Stacey DW: Cyclin D1 serves as a cell cycle regulatory switch in actively proliferating cells. Curr Opin Cell Biol 15: 158-163, 2003.

27. Boonstra J, Rijken P, Humbel B, Cremers F, Verkleij A and van Bergen en Henegouwen P: The epidermal growth factor. Cell Biol Int 19: 413-430, 1995.

28. Cary LA, Han DC and Guan JL: Integrin-mediated signal transduction pathways. Histol Histopathol 14: 1001-1009, 1999.

29. Schlesinger TK, Fanger GR, Yujiri T and Johnson GL: The TAO of MEKK. Front Biosci 3: D1181-D1186, 1998.

30. Xia Z, Dickens M, Raingeaud J, Davis RJ and Greenberg ME: Opposing effects of ERK and JNK-p38 MAP kinases on apoptosis. Science 270: 1326-1331, 1995.

31. Rubinfeld $\mathrm{H}$ and Seger R: The ERK cascade: A prototype of MAPK signaling. Mol Biotechnol 31: 151-174, 2005. 